University of Wollongong

Research Online

Australian Institute for Innovative Materials -

Papers

Australian Institute for Innovative Materials

$1-1-2018$

Free-standing sulfur-polypyrrole cathode in conjunction with polypyrrolecoated separator for flexible Li-S batteries

\author{
Fang Li \\ University of Wollongong, f151@uowmail.edu.au \\ Mohammad Kaiser \\ University of Wollongong, mrk912@uowmail.edu.au \\ Jianmin Ma \\ Hunan University \\ Zaiping Guo \\ University of Wollongong, zguo@uow.edu.au \\ Hua-Kun Liu \\ University of Wollongong, hua@uow.edu.au
}

See next page for additional authors

Follow this and additional works at: https://ro.uow.edu.au/aiimpapers

Part of the Engineering Commons, and the Physical Sciences and Mathematics Commons

Research Online is the open access institutional repository for the University of Wollongong. For further information contact the UOW Library: research-pubs@uow.edu.au 


\title{
Free-standing sulfur-polypyrrole cathode in conjunction with polypyrrole-coated separator for flexible Li-S batteries
}

\begin{abstract}
A free-standing sulfur-polypyrrole cathode and a polypyrrole coated separator were designed for flexible $\mathrm{Li}$-S batteries. The free-standing sulfur-polypyrrole cathode was prepared by directly pasting a sulfurcoated polypyrrole (S@PPy) nanofiber composite on a flexible and conductive polypyrrole (PPy) film. Compared with carbonaceous matrixes, PPy has a strong interaction with polysulfides to mitigate the dissolution of polysulfides due to its unique chain structure and the lone pair electrons in the nitrogen atoms in PPy. In addition, the as-prepared PPy film not only shows excellent mechanical elasticity, but also possesses a rough surface, which can accommodate volume expansion, enhance the adhesion of active materials, and further trap the dissolved polysulfides. Due to the synergistic effect provided by the PPy film, the free-standing sulfur-polypyrrole cathode shows better electrochemical performance than the traditional cathode with S@PPy composite coated on Al foil. In order to further improve the cycling stability of Li-S batteries, a PPy coated separator was prepared, which acts as a fishing net to capture polysulfides and alleviate the shuttle effect, leading to a stable cycling performance. Moreover, the PPy layer coated on commercial separator is much lighter than many other free-standing interlayers reported previously. Considering the flexibility of the free-standing sulfur cathode and PPy coated separator, a softpackaged flexible Li-S battery based on them has been designed and fabricated to power a device consisting of 24 light emitting diode (LED) lights. After repeated bending, the flexible Li-S battery can still maintain good performance, indicating the excellent mechanical flexibility of the designed Li-S battery.

\section{Disciplines}

Engineering | Physical Sciences and Mathematics

\section{Publication Details}

Li, F., Kaiser, M. Rejaul., Ma, J., Guo, Z., Liu, H. \& Wang, J. (2018). Free-standing sulfur-polypyrrole cathode in conjunction with polypyrrole-coated separator for flexible Li-S batteries. Energy Storage Materials, 13 312-322.
\end{abstract}

\section{Authors}

Fang Li, Mohammad Kaiser, Jianmin Ma, Zaiping Guo, Hua-Kun Liu, and Jiazhao Wang 


\title{
Free-standing Sulfur-Polypyrrole Cathode in Conjunction with Polypyrrole-Coated Separator for Flexible Li-S Batteries
}

Fang $\mathrm{Li}^{\mathrm{a}}{ }^{\mathrm{b}}$, Mohammad Rejaul Kaiser ${ }^{\mathrm{a}}$, Jianmin $\mathrm{Ma}^{\mathrm{b}}$, Zaiping Guo ${ }^{\mathrm{c}}$, Huakun Liu ${ }^{\mathrm{a}}$ and Jiazhao Wang $^{\mathrm{a}} *$

${ }^{a}$ Institute for Superconducting and Electronic Materials, University of Wollongong, Wollongong, NSW 2500, Australia

${ }^{b}$ School of Physics and Electronics, Hunan University, Changsha, 40082 China

${ }^{c}$ School of Mechanical, Materials and Mechatronics Engineering, University of Wollongong, North Wollongong, NSW 2500, Australia

\section{Corresponding Author}

*E-mail: jiazhao@uow.edu.au (J. Wang)

\begin{abstract}
A free-standing sulfur-polypyrrole cathode and a polypyrrole coated separator were designed for flexible Li-S batteries. The free-standing sulfur-polypyrrole cathode was prepared by directly pasting a sulfur-coated polypyrrole (S@PPy) nanofiber composite on a flexible and conductive polypyrrole (PPy) film. Compared with carbonaceous matrixes, PPy has a strong interaction with polysulfides to mitigate the dissolution of polysulfides due to its unique chain structure and the lone pair electrons in the nitrogen atoms in PPy. In addition, the asprepared PPy film not only shows excellent mechanical elasticity, but also possesses a rough surface, which can accommodate volume expansion, enhance the adhesion of active materials, and further trap the dissolved polysulfides. Due to the synergistic effect provided by the PPy film, the free-standing sulfur-polypyrrole cathode shows better electrochemical performance than the traditional cathode with S@PPy composite coated on Al foil. In order to further improve the cycling stability of Li-S batteries, a PPy coated separator was prepared, which acts as a fishing net to capture polysulfides and alleviate the shuttle effect, leading to a stable cycling performance. Moreover, the PPy layer coated on commercial separator is much lighter than many other free-standing interlayers reported previously. Considering the
\end{abstract}


flexibility of the free-standing sulfur cathode and PPy coated separator, a soft-packaged flexible Li-S battery based on them has been designed and fabricated to power a device consisting of 24 light emitting diode (LED) lights. After repeated bending, the flexible Li-S battery can still maintain good performance, indicating the excellent mechanical flexibility of the designed Li-S battery.

Keywords: free-standing sulfur-polypyrrole cathode, polypyrrole-coated separator, flexible lithium-sulfur battery

\section{Introduction}

Flexible electronic devices, such as roll-up displays, and implantable and wearable devices, have drawn much attention to promising soft energy storage systems. In order to power these kinds of devices, flexible batteries with both mechanically robust flexibility and high energy density are required. ${ }^{1,2}$ Currently, lithium ion batteries (LIBs) have dominated the portable device industry for decades due to their high energy density, high working voltage, and long lifetime. ${ }^{3,4}$ Due to the restricted volume and weight in modern flexible electronics, however, even state-of-art LIBs based on intercalation chemistry are limited by insufficient capacity. ${ }^{5-7}$ Therefore, exploring new cathode materials with high specific capacity is urgently needed for flexible batteries.

Sulfur, which can react with $\mathrm{Li}^{+}$through a two-electron transfer electrochemistry and exhibits high specific capacity (1675 $\left.\mathrm{mA} \mathrm{h} \mathrm{g}^{-1}\right)$ and high energy density $\left(2600 \mathrm{Wh} \mathrm{kg}^{-1}\right)$, is regarded as a promising cathode material for flexible batteries. Great efforts have been made to date on exploring flexible sulfur cathodes to realize the application of sulfur in flexible devices. Among them, Cheng's group ${ }^{8}$ infiltrated sulfur into flexible graphene foam, prepared using $\mathrm{Ni}$ foam as template, to produce a cathode for Li-S batteries, which showed long-term stability. Peng et al. developed a flexible hybrid cathode containing CMK-3 ordered 
mesoporous carbon, sulfur, and carbon nanotubes (CMK-3@S/CNT) with an aligned and laminated structure to improve the conductivity of the electrode and suppress the shuttle effect. ${ }^{9}$ Niu's group ${ }^{10}$ designed two kinds of soft-packaged and cable-type flexible Li-S batteries based on a free-standing paper-like reduced graphene oxide (rGO)-S film with high flexibility, which showed stable electrochemical performance in the bent state. Although great developments have been made in flexible sulfur cathodes, the flexible matrixes that are used are always nonpolar carbonaceous materials, such as rGO or CNT, which have low binding energy with polar $\operatorname{Li}_{x} \mathrm{~S}(0<x \leq 2) .{ }^{11}$ Compared with carbonaceous materials, polypyrrole (PPy), as a conducting polymer, has many advantages as a cathode matrix for Li$\mathrm{S}$ batteries. First, PPy has strong interactions with intermediate polysulfides due to its unique chain structure and the lone pair electrons of the nitrogen atoms in PPy. ${ }^{12,13}$ Moreover, the redox potential of PPy $\left(2.5 \mathrm{~V}\right.$ vs. $\left.\mathrm{Li}^{-} \mathrm{Li}^{+}\right)$is located in the range of potential windows that are suitable for the Li-S battery. ${ }^{14}$ Therefore PPy not only acts as an electrically conducting agent, but also contributes capacity to the Li-S batteries. ${ }^{15,16}$ Keeping these considerations in mind, we designed a free-standing sulfur-polypyrrole cathode (PPy/S@PPy) by loading sulfur coated polypyrrole (S@PPy) composite on a flexible PPy film. The as-prepared PPy film possesses a rough surface, which can enhance the adhesion of active material and further trap the dissolved polysulfides. Furthermore, the flexible PPy film shows excellent mechanical elasticity, ${ }^{17}$ which can accommodate the volume expansion and prevent the peeling-off and cracking of active materials, which usually occurs in traditional electrodes. Due to the synergistic effect provided by the PPy film, the designed free-standing sulfurpolypyrrole cathode was found to be more suitable for flexible $\mathrm{Li}-\mathrm{S}$ batteries than the traditional electrode with $\mathrm{Al}$ foil as substrate.

Recently, it was demonstrated that the cell configuration also plays a vital role in achieving higher capacity and more stable cyclability. ${ }^{18,}{ }^{19}$ Fang et al. provided an overview of 
promising approaches for realizing Li-S batteries that are suitable for commercialization, in which the electrode architecture, cell engineering, and design parameters are comprehensively discussed for improving the electrochemical performance of the Li-S battery. ${ }^{20}$ Among these promising methods, A. Manthiram and co-workers ${ }^{21}$ inserted a layer of free-standing microporous carbon paper between the cathode and the separator to address the shuttle effect, and they found that the interlayer can effectively decrease the internal resistance and adsorb soluble polysulfides. Inspired by this work, we present a bifunctional separator that is fabricated by directly coating polypyrrole nanofibers on a commercial separator. Compared with the carbonaceous interlayers normally used in $\mathrm{Li}-\mathrm{S}$ batteries, the PPy layer coated on the separator acts as a fishing net to capture dissolved polysulfides to mitigate the shuttle effect, ${ }^{13}$ which can enhance the cycling stability of Li-S batteries. In addition, the PPy layer coated on the separator is much lighter $\left(0.3 \mathrm{mg} \mathrm{cm}^{-2}\right)$ than many free-

standing interlayers fabricated by other groups, ${ }^{22-24}$ which will improve the gravimetric capacity of the whole cell. As a result, the PPy-coated separator will provide Li-S batteries with high capacity and enhanced cycling stability.

In this study, based on the flexibility of the designed free-standing sulfur cathode and the PPy coated separator, a soft-packaged flexible Li-S battery based on them was assembled. Due to the inherent advantages of PPy mentioned above, the newly designed flexible Li-S battery is expected to exhibit stable cycling life and have great practical applications.

\section{Experimental Section}

\subsection{Synthesis of PPy film}

The PPy film was synthesised by an electrodeposition method. The electrodeposition was carried out in a solution containing $0.2 \mathrm{M}$ pyrrole and $0.05 \mathrm{M}$ sodium $p$-toluene sulphonate (p-TSNa). A stainless steel plate was used as the working electrode, a reticulated vitreous 
carbon tube as the counter electrode, and $\mathrm{Ag} / \mathrm{AgCl}$ as the reference electrode. The process was carried out using an automatic battery tester system (Land ${ }^{\circledR}$, China) with a constant current density of $1 \mathrm{~mA} \mathrm{~cm}{ }^{-2}$. After electrodeposition, the PPy free-standing film was easily peeled off from the stainless steel plate and washed with distilled water and ethanol, which was followed by room-temperature drying.

\subsection{Synthesis of PPy nanofiber}

PPy nanofiber was synthesized via an oxidative chemical polymerization method. The pyrrole was distilled before use. In a typical process, $0.72 \mathrm{~g}$ hexadecyl trimethyl ammonium bromide (CTAB) was dissolved in $200 \mathrm{~mL}$ of $1 \mathrm{M} \mathrm{HCl}$ aqueous solution. Then, $0.25 \mathrm{~g}$ sodium $p$-toluene sulfonate and $0.33 \mathrm{~g}$ distilled pyrrole were added into the above solution successively, and the solution was precooled in an ice bath $\left(0-5{ }^{\circ} \mathrm{C}\right)$ maintained for $0.5 \mathrm{~h}$ under constant magnetic stirring. Meanwhile, $1.13 \mathrm{~g}$ ammonium persulfate was dissolved in $20 \mathrm{ml}$ distilled water, which was then dropped into the pyrrole-containing solution. The whole solution was reacted for $12 \mathrm{~h}$ in an ice bath $\left(0-5^{\circ} \mathrm{C}\right)$ under magnetic stirring. After that, the black product was collected by vacuum filtration and washed with $1 \mathrm{M} \mathrm{HCl}$ and distilled water several times, followed by drying at $60{ }^{\circ} \mathrm{C}$ for $12 \mathrm{~h}$ in an oven. Finally, the obtained black powder was denoted as PPy nanofiber.

\subsection{Synthesis of S@PPy composite}

The composite was prepared as follow: $32 \mathrm{mg}$ PPy nanofiber was added into a $50 \mathrm{~mL}$ aqueous solution containing $470 \mu \mathrm{L}$ Triton ${ }^{\circledR} \mathrm{X}-100$ surfactant, followed by sonication for $3 \mathrm{~h}$ to form a stable suspension. A uniform coating of sulfur on the surfaces of the PPy nanofibers was realized by a simple chemical deposition method. Briefly, $0.5 \mathrm{~g} \mathrm{Na}_{2} \mathrm{~S}_{2} \mathrm{O}_{3}$ was first dissolved in $150 \mathrm{ml}$ distilled water, and then the $\mathrm{Na}_{2} \mathrm{~S}_{2} \mathrm{O}_{3}$ solution was added into the asprepared PPy suspension. The mixed solution was stirred at room temperature for $3 \mathrm{~h}$. After 
that, $15 \mathrm{ml} \mathrm{HCl}(0.5 \mathrm{M})$ was slowly added at a rate of $0.1 \mathrm{~mL} \mathrm{~min}^{-1}$. After the reaction, the assynthesized S@PPy composite was collected by vacuum filtration and washed with distilled water several times to remove residue salts and impurities. Finally, the obtained sample was dried at $50{ }^{\circ} \mathrm{C}$ overnight.

\subsection{Fabrication of CNT free-standing film}

The fabrication of the single-walled (SW) CNT free-standing film was exactly the same as in our previous work. ${ }^{25} 15 \mathrm{mg}$ of SWCNT with $500 \mathrm{ml}$ de-ionized water were poured into a beaker, and $500 \mathrm{mg}$ of Triton-X100 surfactant was added. Then, the solution was probe sonicated for $1 \mathrm{~h}$ with a $2 \mathrm{~s}$ pause time, followed by vacuum filtration and washing with deionized water and ethanol. The polytetrafluoroethylene (PTFE) filter paper with the SWCNT layer was dried under vacuum overnight at $60{ }^{\circ} \mathrm{C}$, and finally, the SWCNT film was easily peeled off from the filter paper.

\subsection{Material Characterizations}

For physical and morphological characterization of the composite, X-ray diffraction patterns (XRD, GBC MMA 017) were collected over a $2 \theta$ range of $10^{\circ}-80^{\circ}$ with a scan rate of $2^{\circ}$ $\min ^{-1}$. Raman spectra were collected on a JOBIN YVON HR800 Confocal Raman System with $632.8 \mathrm{~nm}$ diode laser excitation on a 300 lines $\mathrm{mm}^{-1}$ grating at room temperature. The Fourier transform infrared (FTIR) spectrographs were collected using a Nicolet Avatar 360 FTIR Fourier transform infrared spectrometer. X-ray photoelectron spectroscopy (XPS) was conducted on a Vacuum Generator (VG) Scientific ESCALAB 2201XL instrument using Al $\mathrm{K} \alpha \mathrm{X}$-ray radiation and fixed analyser transmission mode. Thermogravimetric analysis (TGA) was performed in air using a SETARAM instrument to estimate the amount of sulfur in the sample. The morphologies of the samples and corresponding element mapping images were examined by field emission scanning electron microscopy (JEOL: FESEM-7500). 


\subsection{Electrochemical Measurements}

The coin cells and soft-packaged batteries were all assembled in an Ar-filled glove box. Lithium metal foil was used as the anode. The PPy film was cut into disks with a diameter of $12 \mathrm{~mm}$ to be used as current collectors. The S@PPy composite slurry was made by mixing 80 wt\% S @PPy with 10 wt\% carbon black and 10 wt\% poly(vinylidene fluoride) (PVDF) binder in N-methyl-2-pyrrolidinone (NMP) solvent. The slurry was spread on the PPy film with a doctor blade. The PPy coated separator was prepared using the same method. The S@PPy electrode and PPy-separator were dried in a vacuum oven at $60{ }^{\circ} \mathrm{C}$ overnight. The mass loading of the sulfur cathode was about $1.4 \mathrm{mg} \mathrm{cm}^{-2}$. The electrolyte was $1 \mathrm{M}$ lithium bis(trifluoromethanesulfonyl)imide (LiTFSI) in 1,3-dioxolane (DOL)/1,2-dimethoxyethane (DME) (1:1 by volume) containing $0.1 \mathrm{M} \mathrm{LiNO}_{3}$ as additive. The soft-packaged Li-S batteries were assembled as follows: the PPy-separator and electrolyte were sandwiched between the PPy/S@PPy electrode and the Li foil, the assembly was then sealed in an Al/polymer soft package, and the same electrolyte as used in the coin cells was injected. The size of the Al/polymer used in the soft-packaged Li-S batteries is $5 \mathrm{~cm} \times 5 \mathrm{~cm}$. The size of both the sulfur cathode and the lithium foil used in the soft-packaged Li-S batteries is $2 \mathrm{~cm} \times$ $2 \mathrm{~cm}$. To verify the practical application of the obtained flexible Li-S battery in flexible devices, it was bent by hand to an angle of about $140^{\circ} 10$ times, and then was used to power the device in the bent state. For electrochemical performance evaluation of the Li-S cell, an automatic battery test system (Land ${ }^{\circledR}$, China) was used at room temperature. Electrochemical impedance spectroscopy (EIS) and cyclic voltammetry (CV) measurements were performed on a Biologic VMP 3 electrochemical workstation over a frequency range of $10 \mathrm{mHz}$ to 100 $\mathrm{kHz}$, and the scan rate was $0.1 \mathrm{mV} \mathrm{s}^{-1}$ within a $1.7 \mathrm{~V}$ to $2.8 \mathrm{~V}$ voltage window. The coin cell charge-discharge testing was carried out with a LAND battery test system at $0.2 \mathrm{C}(1 \mathrm{C}=$ 
$1675 \mathrm{~mA} \mathrm{~g}^{-1}$ ) within the voltage range of $1.7-2.8 \mathrm{~V}$. The soft-packaged Li-S battery was tested at $0.1 \mathrm{C}$.

\section{Results and discussion}

The crystalline structures of the synthesized sulfur, PPy nanofiber, and S@PPy composite were investigated by X-ray diffraction (XRD). As shown in Fig. 1a, all the diffraction peaks of the S@PPy composite match very well with the diffraction lines of sulfur (JCPDS No: 080427), indicating the formation of well-defined crystalline sulfur during the chemical synthesis procedure. PPy only has a broad and weak diffraction peak between $20-30^{\circ}$, however, suggesting an amorphous structure. Raman spectroscopy measurements were also used to confirm the formation of the as-prepared S@PPy composite. Fig. 1b shows the Raman spectra of bare S, PPy nanofiber, and S@PPy composite in the range of 100 to 2000 $\mathrm{cm}^{-1}$. The Raman spectrum of $\mathrm{S}$ displays three main peaks below $500 \mathrm{~cm}^{-1}$. In the case of PPy, the Raman scattering peak located at $1599 \mathrm{~cm}^{-1}$ is ascribed to the $\mathrm{C}=\mathrm{C}$ stretching vibration. The scattering peaks at $1320 \mathrm{~cm}^{-1}$ and $1396 \mathrm{~cm}^{-1}$ are assigned to the ring-stretching mode of PPy. The peak at $1260 \mathrm{~cm}^{-1}$ is assigned to the $\mathrm{N}-\mathrm{H}$ in-plane bending vibration. The peaks at 1043 and $930 \mathrm{~cm}^{-1}$ are assigned to the $\mathrm{C}-\mathrm{H}$ out-of-plane bending vibration. ${ }^{26}$ The Raman spectrum of S@PPy composite shows not only the three characteristic peaks of sulfur below $500 \mathrm{~cm}^{-1}$, but also the typical peaks of PPy between 800 and $1700 \mathrm{~cm}^{-1}$, which confirms that the S@PPy composite contains both elemental sulfur and PPy. X-ray photoelectron spectroscopy (XPS) measurements were performed on the as-prepared PPy nanofiber and S@PPy composite to obtain more detailed information on the surface chemical composition and the chemical states of the elements. The XPS spectrum of PPy indicates the presence of C 1s, N 1s, and $\mathrm{O}$ 1s signals at 285, 399.7, and $532 \mathrm{eV}$, respectively, as shown in

Fig. S1a in the Supporting Information. ${ }^{27}$ In comparison, the spectrum of S@PPy composite shows two additional S 2p and S 2s signals, confirming the successful loading of sulfur on 
PPy nanofibers. The high-resolution spectrum of the S 2p region (Fig. 1c) is deconvoluted into three peaks. The binding energy at $163.6 \mathrm{eV}$ corresponds to $\mathrm{S} 2 \mathrm{p}_{3 / 2}$, which is slightly lower than for elemental sulfur $(164 \mathrm{eV})$, revealing the possible presence of $\mathrm{C}-\mathrm{S}$ species, ${ }^{28,29}$ while the binding energy at $164.9 \mathrm{eV}$ corresponds to $\mathrm{S} 2 \mathrm{p}_{1 / 2}$. The peak at $168.4 \mathrm{eV}$ can be ascribed to the sulfate species formed by the oxidation of sulfur in air and the residual ammonium persulfate. ${ }^{30}$ The binding energy of the $\mathrm{N} 1 \mathrm{~s}$ peak is centred at $399.7 \mathrm{eV}$, which is attributed to pyridinic $\mathrm{N}$ from PPy nanofiber (Fig. S1b). ${ }^{31}$ FTIR experiments (Fig. S1c) were also conducted to confirm the presence of PPy. Compared with the FTIR spectra of PPy and S, the spectrum of S@PPy composite exhibited the clear presence of the vibrational peaks corresponding to PPy, indicating the presence of PPy in the composite. Thermogravimetric analysis (TGA) was carried out in air to determine the content of sulfur in the S@PPy composite. As shown in Fig. 1d, the TGA curves of S@PPy composites consist of three weight loss stages: the first stage from $40^{\circ} \mathrm{C}$ to $130{ }^{\circ} \mathrm{C}$ was ascribed to the evaporation of residual moisture, which is about $4.2 \%$; the second weight loss stage from $130^{\circ} \mathrm{C}$ to $250{ }^{\circ} \mathrm{C}$ was assigned to the sublimation of sulfur, which is $52.6 \%$; and the third stage was the oxidation of PPy nanofiber $\left(250{ }^{\circ} \mathrm{C}-580{ }^{\circ} \mathrm{C}\right)$. Thus, the content of sulfur in the S@PPy composites was found to be $52.6 \%$. From the TGA profile, it is clearly seen that the release temperature of sulfur in S@PPy is slightly lower than that of simple sublimed sulfur. This phenomenon can be ascribed to the uniform dispersion of nanosized sulfur $\mathrm{n}$ S@PPy compared to the bulk-type material in sublimed sulfur. ${ }^{32}$ In addition, the incorporation of PPy fibers will also slightly accelerate the evaporation of sulfur due to the enhanced heat transfer rate. $^{33}$

The morphology of the as-prepared PPy nanofiber and S@PPy composite were investigated by scanning electron microscopy (SEM). As shown in Fig. 2a, the surfaces of the pure PPy nanofibers with a diameter about 50-70 $\mathrm{nm}$ are relatively rough, which can 
increase the surface area and makes the deposition of sulfur on them feasible. After the sulfur coating, the S@PPy composite keeps the nanofiber morphology of PPy with a larger diameter, and the surface becomes relatively smooth, indicating the successful loading of sulfur layer on the PPy nanofiber (Fig. 2b). The corresponding elemental maps of carbon, nitrogen, and sulfur in Fig. 2d-e further confirm the homogeneous distribution of elements in the S@PPy composite. A typical photograph of the as-prepared PPy film is shown in Fig. 3a, which shows excellent flexibility. The free-standing PPy flim can be folded into one fourth of its original size, and could returned to its original appearance without any cracks, while $\mathrm{Al}$ foil cannot recover its original shape, as shown in Fig. S2 and Fig. 3f. Due to the elastic properties of PPy film, it could release mechanical stresses and prevent the peeling-off and cracking of the active material during cycling, which is necessary for a flexible Li-S battery. As shown in Fig. 3b, the PPy film with a thickness of about $10 \mu \mathrm{m}$ shows a rough surface consisting of many nodules, which provides a larger contact area and stronger adhesion with the active material compared with the smooth $\mathrm{Al}$ foil. The adhesion of the active materials to the PPy film and Al foil are shown by cross-sectional SEM images of the electrodes (Fig. 3cd). The compact and homogenous S@PPy active material tightly adheres to the PPy film (Fig. 3c), while there is a gap between the S@PPy nanofiber material and the Al foil current collector because of the weak adhesion between the smooth Al foil and the active materials (Fig. 3d), which will dramatically increase the internal impedance and lead to sluggish redox reactions. In addition, the PPy/S@PPy electrode could keep its integrity, and there is no cracking or exfoliation of the active materials after bending, which can ascribed to the mechanical elasticity of the PPy film (Fig. 3e), while the Al/S@PPy electrode forms cracks and the electrical connectivity is broken after bending (Fig. 3f). On the other hand, the weight of the PPy film is only one third that of the Al foil (Table S1), which will increase the gravimetric capacity and energy density of the whole cell. As such, the free-standing 
PPy/S@PPy electrode is more suitable as a flexible Li-S cathode than the A1/S@PPy electrode.

To verify the effects of the PPy substrate on the electrochemical performance, a series of electrochemical measurements were carried out to compare it with conventional Al foil substrate. The same amount of S@PPy nanofiber was coated on Al foil and PPy film to make cathodes for Li-S batteries (denoted as Al/S@PPy and PPy/S@PPy, respectively). Cyclic voltammetry (CV) measurements were conducted on the Al/S@PPy and PPy/S@PPy electrodes over the voltage range from $1.7 \mathrm{~V}-2.8 \mathrm{~V}$ at a scanning rate of $0.1 \mathrm{mV} \mathrm{s}^{-1}$ (Fig. S3a and b). Both electrodes show two typical cathodic peaks and one broad anodic peak. The first cathodic peak located at about $2.3 \mathrm{~V}$ represents the reduction of sulfur to soluble long-chain polysulfides $\left(\mathrm{Li}_{2} \mathrm{~S}_{\mathrm{n}}, 2<n \leq 8\right)$, and the second cathodic peak at $\sim 2.0 \mathrm{~V}$ is due to the further reduction of soluble polysufides to lithium sulfides $\left(\mathrm{Li}_{2} \mathrm{~S}_{2} / \mathrm{Li}_{2} \mathrm{~S}\right) .{ }^{34}$ At around $2.5 \mathrm{~V}$, the anodic peak reveals the conversion from lithium sulfides to lithium polysulfides, and finally to sulfur. ${ }^{35,36}$ The $\mathrm{CV}$ peaks for the Al/S@PPy electrode (Fig. S3b) show a lower reduction potential and higher oxidation potential than those for PPy/S@PPy electrode, indicating higher voltage hysteresis and sluggish kinetics. ${ }^{37}$ Electrochemical impedance spectroscopy (EIS) measurements were used to characterize the internal resistance and charge-transfer process. The Nyquist plots of the PPy/S@PPy and Al/S@PPy electrodes before cycling are shown in Fig. 4a, which are both composed of a depressed semicircle from the high frequency region to the mid-frequency region and an inclined line in the low frequency region, which are ascribed to the charge-transfer resistance $\left(R_{\mathrm{ct}}\right)$ and the mass-transfer process, ${ }^{38}$ respectively. The Nyquist plots were analyzed and fitted by an equivalent circuit model (inset in Fig. 4a). In the equivalent circuit model, $R_{s}, R_{c t}, Z_{w}$, and $C_{d l}$ represents the impedance of the electrolyte, the charge transfer resistance, the Warburg impedance, and the capacitance of the electrical double layer, respectively. ${ }^{39}$ According to the fitting results 
(Table S2), the charge-transfer resistance of the free-standing PPy/S@PPy electrode (425.6 $\Omega)$ is slightly lower than that of the Al/S@PPy electrode (556.8 $\Omega$ ). The conductivity of the pure PPy film is $19.23 \mathrm{~S} \mathrm{~cm}^{-1}$, however, which is much lower than that of Al foil $\left(3.7 \times 10^{5} \mathrm{~S} \mathrm{~cm}^{-}\right.$ $\left.{ }^{1}\right)$. The lower charge-transfer resistance would be ascribed to the rough surface of the PPy film and tight adhesion to the S@PPy composite. The first charge and discharge voltage profiles of the Li-S batteries with PPy/S@PPy and Al/S@PPy cathodes within the potential window of 1.7-2.8 V vs. $\mathrm{Li}^{+} / \mathrm{Li}$ were collected, as shown in Fig. 4b. The discharge curves show two plateaus, representing two reduction processes, while the charge curves have one plateau, which matches well with the CV curves. It should be noted that the PPy/S@PPy cathode shows lower polarization $(229 \mathrm{mV})$ than the Al/S@PPy cathode $(249 \mathrm{mV})$, which is ascribed to the kinetically efficient redox reaction process with a small barrier for the PPy/S@PPy cathode..$^{40}$

To further clarify the merits of free-standing PPy film as current collector, the cycling performance of Li-S batteries with PPy film current collector was investigated for comparison with cells using porous CNT current collector and conventional Al foil at a current density of $0.2 \mathrm{C}$, as shown in Figure 4c. The same amount of S@PPy nanofiber active materials were coated on PPy film, CNT film, and Al foil, respectively, to make electrodes for Li-S batteries (denoted as PPy/S@PPy, CNT/S@PPy, and Al/S@PPy). The PPy/S@PPy and CNT/S@PPy electrodes both showed higher specific capacity than that of Al/S @PPy due to the porous structures of PPy and CNT film, which can store more electrolyte and enhance the contact between the active materials and the current collector, improving the utilization of active materials. On the other hand, theCNT/S@PPy electrode showed higher initial specific capacity (1081 mA h g-1) than the PPy/S@PPy electrode (985 mA h g-1), which can be ascribed to the $3 \mathrm{D}$ conductive $\mathrm{CNT}$ scaffold, which could provide interconnected ion and electron pathways and good electrical contact with the active materials. The PPy/S@PPy 
electrode retained a capacity of $653 \mathrm{~mA} \mathrm{~h} \mathrm{g-1} \mathrm{after} 100$ cycles, however, with a capacity retention rate of $66.3 \%$, while the discharge capacity of the CNT/S@PPy electrode decreased to $617 \mathrm{~mA} \mathrm{~h} \mathrm{g-1} \mathrm{after} 100$ cycles, with lower capacity retention of $57 \%$. This result indicates that the PPy/S@PPy electrode has better cycling stability than the CNT/S@PPy electrode, which demonstrates that the PPy film features stronger adsorption of polar polysulfides than the nonpolar CNT film due to its unique chain structure and inter- and/or intra-chain bonding with polysulfides. Therefore, the superior electrochemical performance of the PPy/S@PPy electrode is attributable to the synergistic effect provided by the rough and elastic PPy flim, including the strong adhesion between it and the active materials, trapping dissolved polysulfides and accommodating volume expansion. To further confirm the role of the PPy film in improving electrochemical performance, the cell was disassembled after 100 cycles. Fig. $4 \mathrm{~d}$ presents digital photographs of the cycled electrodes and corresponding separators. A small amount of active materials was exfoliated from the Al/S@PPy electrode (Fig. 4d-1) after cycling. In contrast, the S@PPy nanofibers on PPy flim showed no obvious exfoliation or changes (Fig. 4d-2), indicating good elasticity of the PPy film, which can accommodate the volume expansion and prevent peeling-off of the active materials during cycling. The separator from the Li-S battery with Al/S@ PPy electrode showed a yellowish color (Fig. 4d3), indicating the diffusion of polysulfides through the separator, leading to the shuttle effect and low active material utilization. The separator from the Li-S battery with PPy/S@PPy electrode, however, remained relatively clean with no yellowish color (Fig. 4d-4), which means that the PPy film could act as a further barrier to polysulfide migration. All the results demonstrate that the PPy film could not only maintain the integrity of the electrode during cycling, but also limited the dissolution of polysulfide, which improved the active material utilization and guaranteed a stable cycling performance. 
Although the PPy/S@PPy cathode exhibits better cycling performance than CNT/S@PPy and Al/S@PPy electrodes, capacity decay with increasing cycle number still remains a problem, obstructing its practical application. In order to increase the specific capacity and cycling stability, a PPy nanofiber coated separator (PPy-separator) was used in our following experiments. The separator was prepared by a simple doctor blade method, which is simple and cost-effective compared with preparing free-standing interlayers. Most importantly, the PPy layer on the separator can effectively block the diffusion of polysulfides across the separator to the $\mathrm{Li}$ anode, due to its unique chain structure and inter- and/or intra-chain bonding with polysulfides, which will greatly improve the cycling stability.

Photographs of the commercial separator and the as-prepared PPy-separator are shown in Fig. 5a. A homogeneous PPy layer was coated on the commercial separator, and the PPyseparator exhibits good flexibility (as shown in the inset photograph of Fig. 5a). Fig. 5b presents an SEM image of the commercial separator, which displays a highly porous structure with pore size of several hundred nanometres, which the intermediate polysulfides can easily pass through. In contrast, the cross-sectional image of the PPy-separator in Fig. 5c shows that the PPy layer with a thickness about $5 \mu \mathrm{m}$ is closely coated on the commercial separator, which not only can improve the conductivity of the cell, but also works as an effective reservoir for dissolved polysulfides to mitigate the shuttle effect. The PPy-separator shows a loose nanofiber structure (Fig. 5d), which forms a woven-like textile that can act as a fishing net for capturing polysulfides. At the same time, the loose structure ensures that the liquid electrolyte penetrates easily into the whole cell to guarantee rapid redox reactions. The electrochemical properties of a cell with the PPy-separator were investigated, and a cell with a free-standing CNT interlayer was also tested for comparison.

To analyze the impact of the PPy-separator on the performance of $\mathrm{Li}-\mathrm{S}$ batteries, impedance analysis was performed on $\mathrm{Li}-\mathrm{S}$ batteries with commercial separator, PPy- 
separator, and a CNT interlayer before cycling (Fig. 6a). Compared with the battery with the commercial separator, the charge transfer resistances of the Li-S batteries with the PPyseparator or CNT interlayer both dramatically decrease (Table S2). The conductive interlayer between the cathode and the separator acts as an upper current collector, which can maintain contact with the active material on the nanoscale and allows electrons to flow freely through the cathode, reducing the effective resistance of the highly insulating sulfur cathode and accelerating the kinetics of the electrochemical reactions. Moreover, the PPy-separator shows a smaller charge transfer resistance than the CNT interlayer, which can be attributed to the tight contact between the PPy layer and the separator. Fig. $6 \mathrm{~b}$ and $6 \mathrm{c}$ shows the cycling and rate performance of the Li-S batteries with PPy/S@PPy, PPy/S@PPy+PPy-separator, and the PPy/S@PPy+CNT interlayer structure. Compared with the Li-S batteries with commercial separator, the cycling and rate performances of the Li-S batteries with the PPy-separator and CNT interlayer are better, indicating that the conductive layers between the cathode and the separator can effectively capture polysulfides and improve sulfur utilization. As shown in Fig. $6 \mathrm{~b}$, the initial discharge capacity of the Li-S battery with the PPy/S@PPy+PPy-separator structure is $1236 \mathrm{~mA} \mathrm{~h} \mathrm{~g}^{-1}$ at $0.2 \mathrm{C}$, and the battery still retains discharge capacity of 1073 $\mathrm{mA} \mathrm{h} \mathrm{g} \mathrm{g}^{-1}$ after 200 cycles, with a capacity retention rate of about $86.8 \%$. On the other hand, the initial discharge capacity of the Li-S battery with the PPy/S@PPy+CNT interlayer structure is $1158 \mathrm{~mA} \mathrm{~h} \mathrm{~g}^{-1}$, and the capacity after 200 cycles is $907 \mathrm{~mA} \mathrm{~h} \mathrm{~g}^{-1}$, which is much lower than that of the PPy/S@PPy+PPy-separator structure. In addition, the Li-S battery with PPy-separator delivers higher discharge capacity than that with the CNT interlayer at all current densities (Fig. 6c), which indicates that the PPy features stronger adsorption of polysulfides than the CNT interlayer due to its unique chain structure and inter- and/or intrachain bonding with polysulfides. The CV curves of a battery with PPy/S@PPy+PPyseparator structure show two cathodic peaks and one broad anodic peak (Fig. S3d), and the 
peak intensities and positions are almost the same from the second to the fifth cycle, which indicates the good cycling stability of this system. The CV curves of a battery with the $\mathrm{PPy} / \mathrm{S} @ \mathrm{PPy}+\mathrm{CNT}$ interlayer structure show a slightly lower reduction potential and a slightly higher oxidation potential (Fig. S3c), and the integral areas are decreased with the increasing cycle number, which suggests capacity fading during cycling. The galvanostatic discharge profiles of a battery with the PPy/S@PPy+PPy-separator structure at different current densities (from 0.1 C to $1 \mathrm{C}$ ) consist of two plateaus (Fig. 6d), even at a very high current rate. The plateaus are flat and stable with a relatively low polarisation of $151 \mathrm{mV}$ at $0.1 \mathrm{C}$, which suggests a kinetically efficient reaction process with a small barrier. In contrast, the discharge potential decreases, and the charge potential increases for the Li-S batteries with PPy/S@PPy+CNT interlayer, PPy/S@PPy, and Al/S@PPy structures, resulting in higher voltage hysteresis $(166,178$, and $197 \mathrm{mV}$ at $0.1 \mathrm{C}$, respectively) (Fig. S4). In addition, the weight of the PPy layer coated on the separator is much lighter $\left(0.3 \mathrm{mg} \mathrm{cm}^{-2}\right)$ than that of the CNT interlayer $\left(1.03 \mathrm{mg} \mathrm{cm}^{-2}\right.$ ) (Table $\left.\mathrm{S} 1\right)$, which will further improve the gravimetric capacity of the whole cell.

To understand how the PPy-coated separator suppresses the polysulfide diffusion, the coin cells were disassembled in a glove box after 50 cycles. A cross-sectional SEM image of the cycled PPy-coated separator and the corresponding element mapping are shown in Fig. 7a. The PPy layer maintains its original structure and is still firmly adhered to the separator. The mapping image of sulfur indicates that the dissolved polysulfides are mainly trapped within the PPy layer before they pass through the separator because only very weak sulfur signals are detected on the commercial separator. As further evidence, SEM images of the Li anode surface after 50 cycles are shown in Fig. 7c and d. Compared with the fresh metallic Li (Fig. 7b), the Li anode from the cell with the PPy/S@PPy cathode and the commercial separator exhibits a severely damaged surface caused by the reaction of lithium with long chain lithium 
polysulfides $\left(\mathrm{Li}_{2} \mathrm{~S}_{\mathrm{n}} 2<n \leq 8\right)$. In contrast, the Li anode from the cell with the PPy/S@PPy electrode and the PPy-separator shows a relatively smooth surface, which indicates that the polysulfides are mainly trapped on the cathode side rather than travelling to the anode side. This phenomenon illustrates the superior ability of the PPy-separator to suppress the shuttle effect and prevent the corrosion of the $\mathrm{Li}$ anode during cycling. Table $\mathrm{S} 3$ compares the electrochemical performance of this Li-S battery with the PPy/S@PPy+PPy-separator structure with some Li-S batteries reported previously using polypyrrole as an adsorption medium for polysulfides. ${ }^{30,41-44}$ The mass loading of active material is comparable in these papers. The specific capacities of the designed Li-S battery at different C-rates are quite remarkable and higher than those observed in previously reported composite electrodes. This is due to the unique PPy film substrate and the PPy coated separator as a polysulfide reservoir.

Fig. 8a and b clearly illustrates the advantages of the designed structure compared with the traditional electrode structure. First, the flexible and conductive PPy film has a rough surface, which can enhance adhesion of the active materials and trap polysulfides, resulting in low contact resistance and stable cycling performance. Second, PPy is an active material, which can contribute capacity during cycling, as shown in Fig. S5a and b. Moreover, the PPy film could maintain its integrity even after 100 cycles (Fig. S5d) due to its elastic property, which gives it electrochemical stability during cycling. Finally, the PPy layer coated on commercial separator not only acts as a reservoir for capturing polysulfides for reutilization, inhibiting the shuttle effect, but also acts as an upper current collector to facilitate electron transfer. As a result, the designed Li-S battery shows excellent cycling and rate performance.

In order to verify the potential application of the cell with the PPy/S@PPy cathode and PPy-separator in flexible Li-S batteries, soft-packaged Li-S batteries consisting of the freestanding PPy/S@PPy film cathode, the PPy-coated separator, and lithium foil anode were assembled in a glove box. Fig. 9a shows the initial charge-discharge curves of a soft- 
packaged battery before and after bending. The charge-discharge curves before and after bending are similar, indicating that the bending operation makes no difference to the redox reactions of sulfur. The cycling performance of the soft-packaged Li-S battery is shown in Fig. 9b. It delivers an initial discharge capacity of $1064 \mathrm{~mA} \mathrm{~h} \mathrm{~g}^{-1}$ at $0.1 \mathrm{C}$. After 20 cycles, the discharge capacity is still maintained at $848 \mathrm{~mA} \mathrm{~h} \mathrm{~g}^{-1}$ with capacity retention of $79.7 \%$. After that, the flexible battery was bent 10 times, and charged and discharged under the same conditions. It still could retain almost the same capacity compared with that before bending, which indicates that the electrochemical behaviour of the flexible Li-S battery was only slightly influenced by repeated bending. EIS measurements were conducted before and after repeated bending (Fig. 9c). The results indicated that, after the bending, the conductivity of the cell was slightly lower than that of the unbent cell, which can be ascribed to the loose contact between the $\mathrm{Li}$ anode and the PPy/S@PPy cathode during the repeated bending process. SEM images of the free-standing PPy/S@PPy cathode before and after the bending are shown in Fig. 9d. No cracks were detected in the electrode around the bent area after the bending test. This suggests that the electrode is resistant to repeated bending. To demonstrate the practical application of the designed flexible Li-S battery, the soft-packaged battery was used to light up a "UOW" device containing 24 white light-emitting diodes (LEDs) (Fig. 9e and f). The LEDs could still be well lit when the soft-packaged Li-S battery was bent to an angle of about $140^{\circ}$. In short, the designed flexible Li-S battery shows excellent mechanical and electrochemical properties during a rough bending treatment, which is attributed to the novel free-standing PPy/S @PPy cathode and the PPy-coated separator. First, the rough and elastic PPy film will enhance the adhesion of the active material and relieve the mechanical stress during bending. Second, the PPy-coated separator could efficiently trap dissolved polysulfides to improve the utilization of the active material, leading to superior 
electrochemical performance. All these results show its great potential for powering flexible electronics.

\section{Conclusions}

In summary, a free-standing sulfur-PPy cathode and a PPy nanofiber coated separator were designed for flexible Li-S batteries. The as-prepared PPy film not only has a rough surface, which can enhance adhesion of the active materials and trap dissolved polysulfides, but also possesses elastic properties, which can accommodate the volume expansion and maintain the integrity of electrode during cycling. On the other hand, the PPy-separator not only acts as a reservoir for soluble lithium polysulfides, but also acts as an upper current collector to accelerate the kinetics of the electrochemical reactions. Moreover, PPy is electrochemically active and could contribute capacity to Li-S batteries. Benefiting from the above advantages, the flexible Li-S battery can deliver an initial discharge capacity of $1064 \mathrm{~mA} \mathrm{~h} \mathrm{~g}^{-1}$ and retains a capacity of $848 \mathrm{~mA} \mathrm{~h} \mathrm{~g}^{-1}$ after 20 cycles at $0.1 \mathrm{C}$. After repeated bending for 10 times, the capacity remains almost the same. In addition, the soft-packaged Li-S battery could power a device containing 24 white LEDs, both before and after bending, indicating its great potential application in flexible electronics. We believe that this flexible electrode structure may provide guidance for fabricating high energy, flexible electrochemical energy-storage devices.

\section{Acknowledgements}

The authors gratefully appreciate the financial support provided by an Australian Research Council (ARC) Linkage Project (LP160100914). The authors would like to thank the Australian National Fabrication Facility - Materials Node and the UOW Electron Microscopy Centre for equipment use. Many thanks also go to Dr. Tania Silver for critical reading of the manuscript.

\section{References}


1. $\quad$ H. Nishide and K. Oyaizu, Science, 2008, 319, 737-738.

2. $\quad$ G. Zhou, F. Li and H.-M. Cheng, Energ Environ Sci, 2014, 7, 1307-1338.

3. J.-M. Tarascon and M. Armand, Nature, 2001, 414, 359-367.

4. V. Etacheri, R. Marom, R. Elazari, G. Salitra and D. Aurbach, Energy \& Environmental Science, 2011, 4, 3243-3262.

5. J. Cheng, B. Wang, H. L. Xin, C. Kim, F. Nie, X. Li, G. Yang and H. Huang, J Mater Chem A, 2014, 2, 2701-2707.

6. L. Hu, H. Wu, F. La Mantia, Y. Yang and Y. Cui, ACS nano, 2010, 4, 5843-5848.

7. J. Liu, K. Song, P. A. van Aken, J. Maier and Y. Yu, Nano Lett, 2014, 14, 2597-2603.

8. G. Zhou, L. Li, C. Ma, S. Wang, Y. Shi, N. Koratkar, W. Ren, F. Li and H.-M. Cheng, Nano Energy, 2015, 11, 356-365.

9. Q. Sun, X. Fang, W. Weng, J. Deng, P. Chen, J. Ren, G. Guan, M. Wang and H. Peng, Angewandte Chemie, 2015, 127, 10685-10690.

10. J. Cao, C. Chen, Q. Zhao, N. Zhang, Q. Lu, X. Wang, Z. Niu and J. Chen, Adv Mater, 2016, 28, 9629-9636.

11. G. Zheng, Q. Zhang, J. J. Cha, Y. Yang, W. Li, Z. W. Seh and Y. Cui, Nano Lett, 2013, 13, 1265-1270.

12. L. Xiao, Y. Cao, J. Xiao, B. Schwenzer, M. H. Engelhard, L. V. Saraf, Z. Nie, G. J. Exarhos and J. Liu, Adv Mater, 2012, 24, 1176-1181.

13. W. Li, Q. Zhang, G. Zheng, Z. W. Seh, H. Yao and Y. Cui, Nano Lett, 2013, 13, 5534-5540.

14. J. Wang, C. O. Too, D. Zhou and G. G. Wallace, J Power Sources, 2005, 140, 162167.

15. P. Xin, B. Jin, H. Li, X. Lang, C. Yang, W. Gao, Y. Zhu, W. Zhang, S. Dou and Q. Jiang, ChemElectroChem, 2017, 4, 115-121.

16. X. Liang, M. Zhang, M. R. Kaiser, X. Gao, K. Konstantinov, R. Tandiono, Z. Wang, H.-K. Liu, S.-X. Dou and J. Wang, Nano Energy, 2015, 11, 587-599.

17. M. D. Hager, P. Greil, C. Leyens, S. van der Zwaag and U. S. Schubert, Adv Mater, 2010, 22, 5424-5430.

18. S.-H. Chung and A. Manthiram, The journal of physical chemistry letters, 2014, 5, 1978-1983.

19. C.-H. Chang, S.-H. Chung and A. Manthiram, J Mater Chem A, 2015, 3, 1882918834.

20. R. Fang, S. Zhao, Z. Sun, D. W. Wang, H. M. Cheng and F. Li, Adv Mater, 2017.

21. Y.-S. Su and A. Manthiram, Nat Commun, 2012, 3, 1166.

22. Y.-S. Su and A. Manthiram, Chem Commun, 2012, 48, 8817-8819.

23. K. Zhang, F. Qin, J. Fang, Q. Li, M. Jia, Y. Lai, Z. Zhang and J. Li, Journal of Solid State Electrochemistry, 2014, 18, 1025-1029.

24. T.-G. Jeong, Y. H. Moon, H.-H. Chun, H. S. Kim, B. W. Cho and Y.-T. Kim, Chem Commun, 2013, 49, 11107-11109.

25. M. R. Kaiser, J. Wang, X. Liang, H.-K. Liu and S.-X. Dou, Journal of Power Sources, 2015, 279, 231-237.

26. I. Sultana, M. Rahman, S. Li, J. Wang, C. Wang, G. G. Wallace and H.-K. Liu, Electrochim Acta, 2012, 60, 201-205.

27. L. Wang, S. Dou, J. Xu, H. K. Liu, S. Wang, J. Ma and S. X. Dou, Chem Commun, 2015, 51, 11791-11794.

28. C. Zu and A. Manthiram, Advanced Energy Materials, 2013, 3, 1008-1012.

29. L. Zhang, L. Ji, P.-A. Glans, Y. Zhang, J. Zhu and J. Guo, Physical Chemistry Chemical Physics, 2012, 14, 13670-13675. 
30. Y. Dong, S. Liu, Z. Wang, Y. Liu, Z. Zhao and J. Qiu, Nanoscale, 2015, 7, 75697573.

31. J. Shao, X. Li, L. Zhang, Q. Qu and H. Zheng, Nanoscale, 2013, 5, 1460-1464.

32. X. Li, M. Rao, H. Lin, D. Chen, Y. Liu, S. Liu, Y. Liao, L. Xing, M. Xu and W. Li, J Mater Chem A, 2015, 3, 18098-18104.

33. C. Wang, W. Wan, J.-T. Chen, H.-H. Zhou, X.-X. Zhang, L.-X. Yuan and Y.-H. Huang, J Mater Chem A, 2013, 1, 1716-1723.

34. X. Ji, K. T. Lee and L. F. Nazar, Nature materials, 2009, 8, 500-506.

35. X. Ji and L. F. Nazar, J Mater Chem, 2010, 20, 9821-9826.

36. Y. Yang, G. Zheng and Y. Cui, Chem Soc Rev, 2013, 42, 3018-3032.

37. G. Zheng, Y. Yang, J. J. Cha, S. S. Hong and Y. Cui, Nano Lett, 2011, 2011, 4462.

38. G. Zhou, E. Paek, G. S. Hwang and A. Manthiram, Nat Commun, 2015, 6.

39. N. A. Canas, K. Hirose, B. Pascucci, N. Wagner, K. A. Friedrich and R. Hiesgen, Electrochim Acta, 2013, 97, 42-51.

40. G. Zhou, S. Pei, L. Li, D. W. Wang, S. Wang, K. Huang, L. C. Yin, F. Li and H. M. Cheng, Adv Mater, 2014, 26, 625-631.

41. N. Nakamura, T. Yokoshima, H. Nara, T. Momma and T. Osaka, J Power Sources, 2015, 274, 1263-1266.

42. W. Qin, B. Fang, S. Lu, Z. Wang, Y. Chen, X. Wu and L. Han, Rsc Adv, 2015, 5, 13153-13156.

43. Y. Fu and A. Manthiram, Rsc Adv, 2012, 2, 5927-5929.

44. Y. Xie, H. Zhao, H. Cheng, C. Hu, W. Fang, J. Fang, J. Xu and Z. Chen, Applied Energy, 2016, 175, 522-528. 

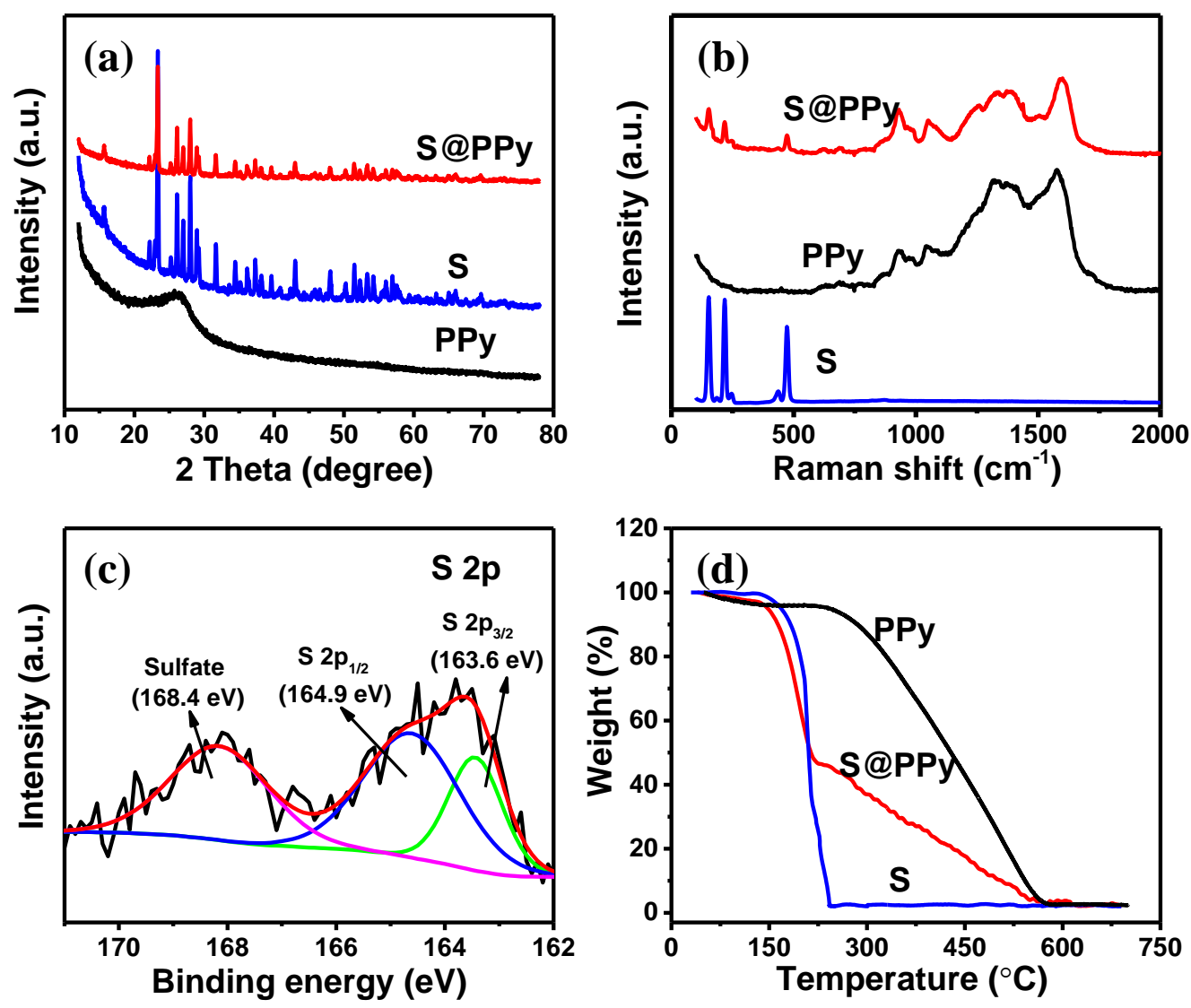

Fig. 1. (a) XRD patterns of synthesized sulfur, PPy nanofiber, and S@PPy composite; (b) Raman spectra of bare S, prepared PPy nanofiber, and S@PPy composite; (c) High resolution S 2p XPS spectrum of S@PPy composite; (d) TGA curves of synthesized sulfur, PPy nanofiber, and S@PPy composite in air. 

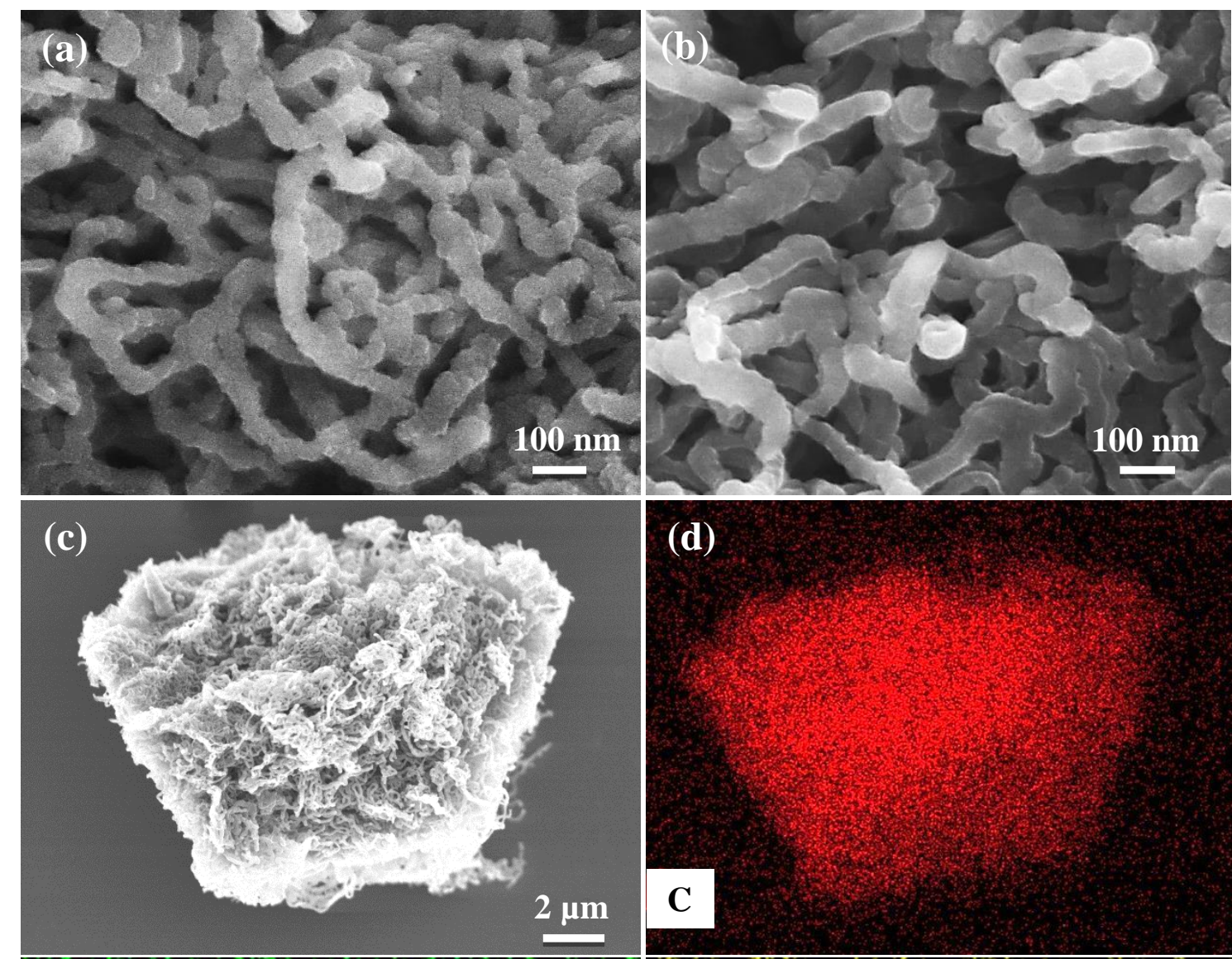

\section{(d)}
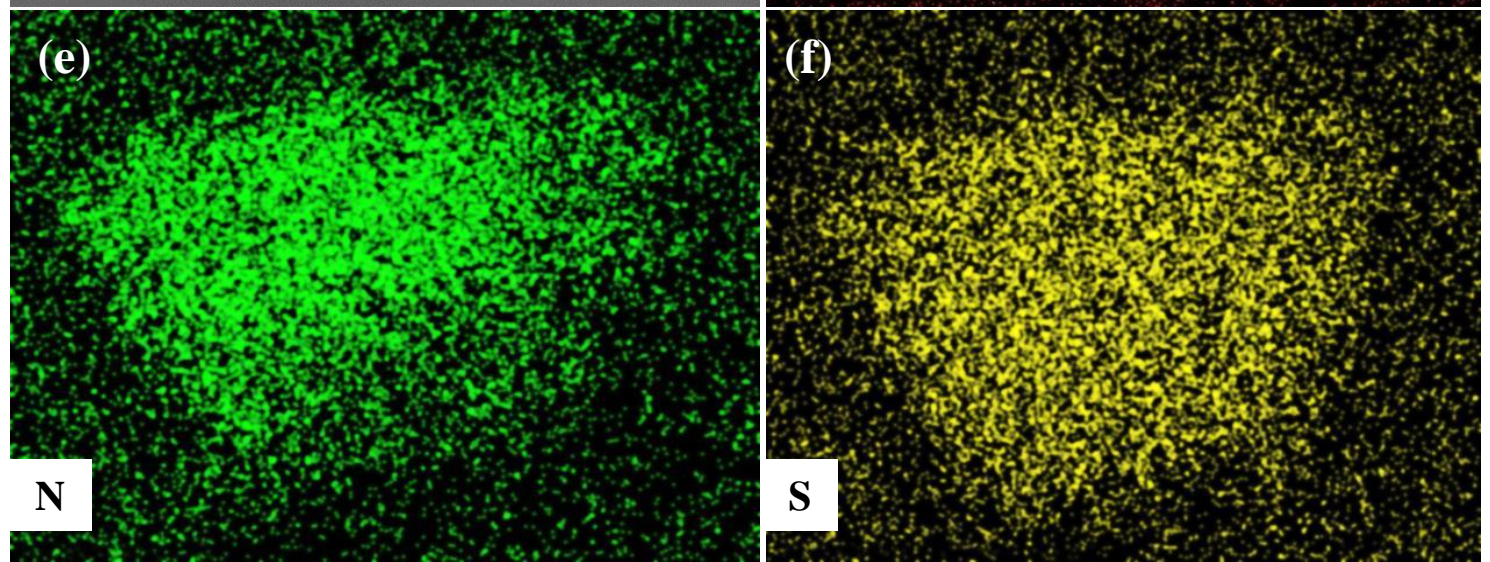

Fig. 2. SEM images of (a) PPy nanofiber and (b) S@PPy composite; (c) Low-resolution SEM image and corresponding elemental mapping showing the uniform distribution of (d) C, (e) N, and (f) S elements in S@PPy composite. 


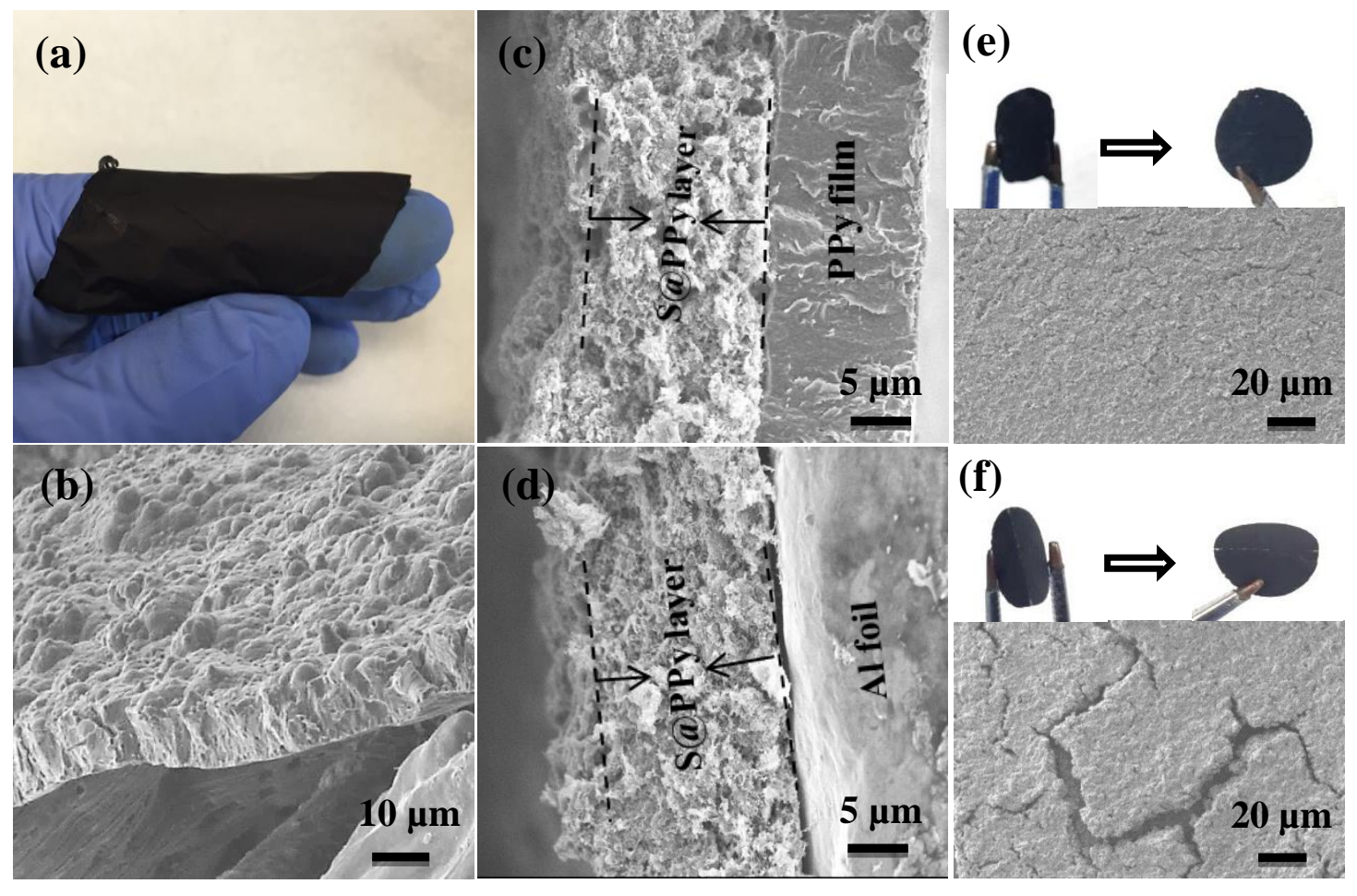

Fig. 3. (a) Digital photograph and (b) SEM image of as-prepared PPy film; SEM crosssectional images of (c) PPy/S@PPy and (d) Al/S@PPy electrodes; (e) Digital photographs of PPy/S@PPy electrode under and after bending, and SEM image of PPy/S@PPy electrode after bending; (f) Digital photographs of Al/S@PPy electrode under and after bending, and SEM image of Al/S@PPy electrode after bending. 

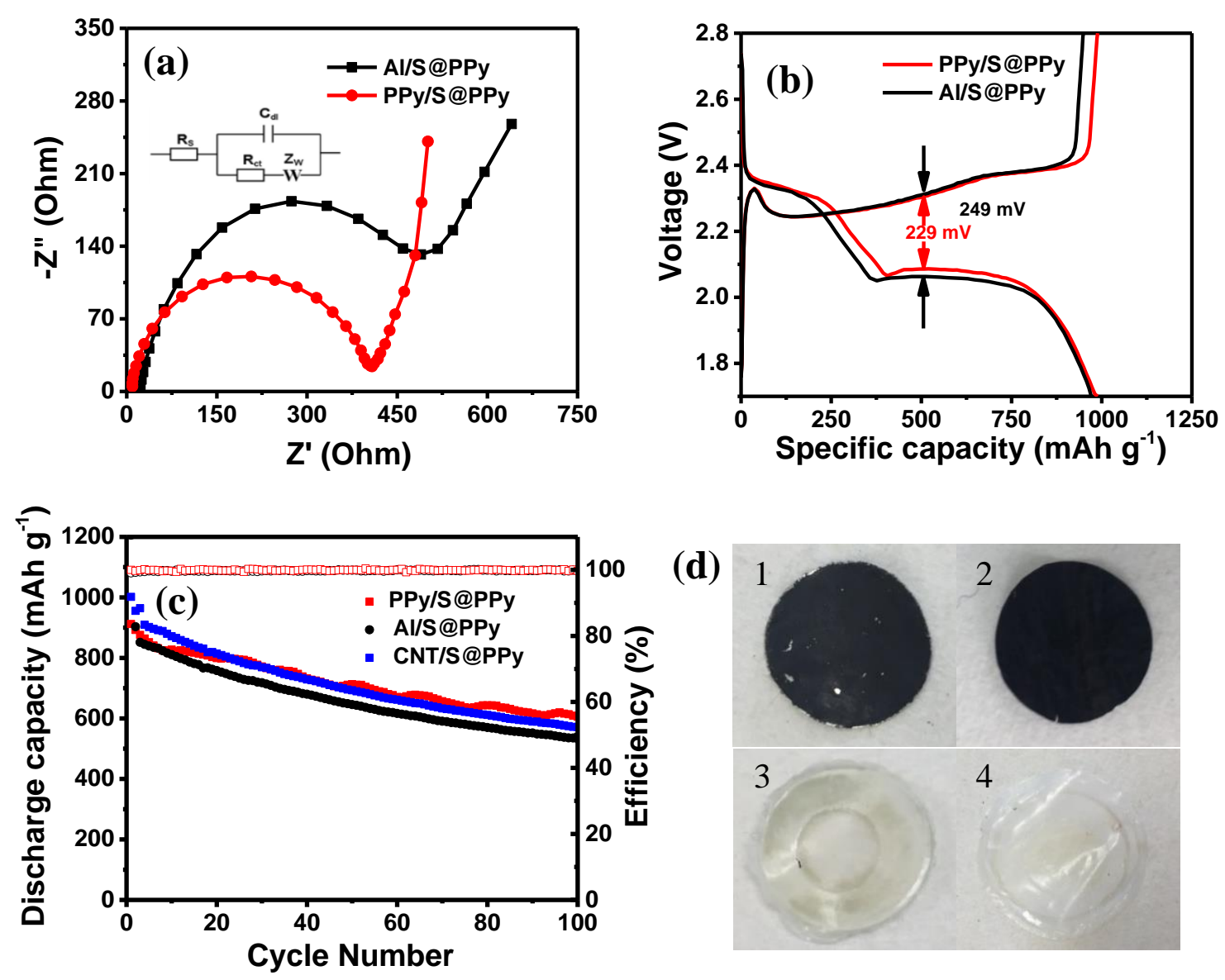

(d)

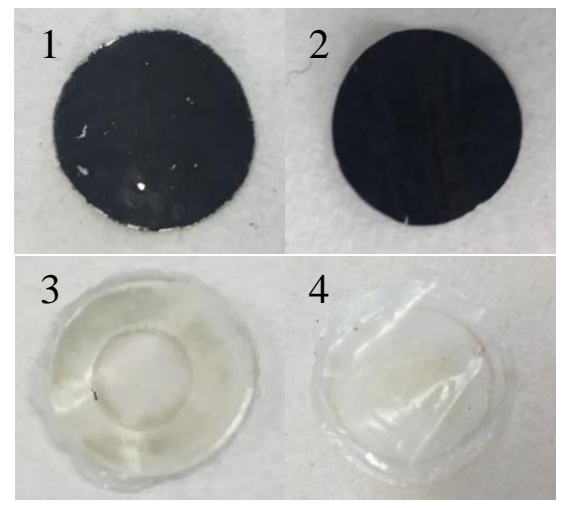

Fig. 4. (a) Nyquist plots of the PPy/S@PPy and Al/S@PPy electrodes before cycling (inset: equivalent circuit); (b) Galvanostatic charge-discharge profiles of PPy/S@PPy and Al/S@PPy electrodes; (c) Cycling performances of Li-S batteries with PPy/S@PPy, CNT/S@PPy, and Al/S@PPy cathodes at 0.2 C for 100 cycles; (d) Photographs of cycled (1) Al/S@PPy electrode and (2) PPy/S@PPy electrode, and photographs of separators disassembled from Li-S batteries with (3) Al/S@PPy electrode and (4) PPy/S@PPy electrode. 


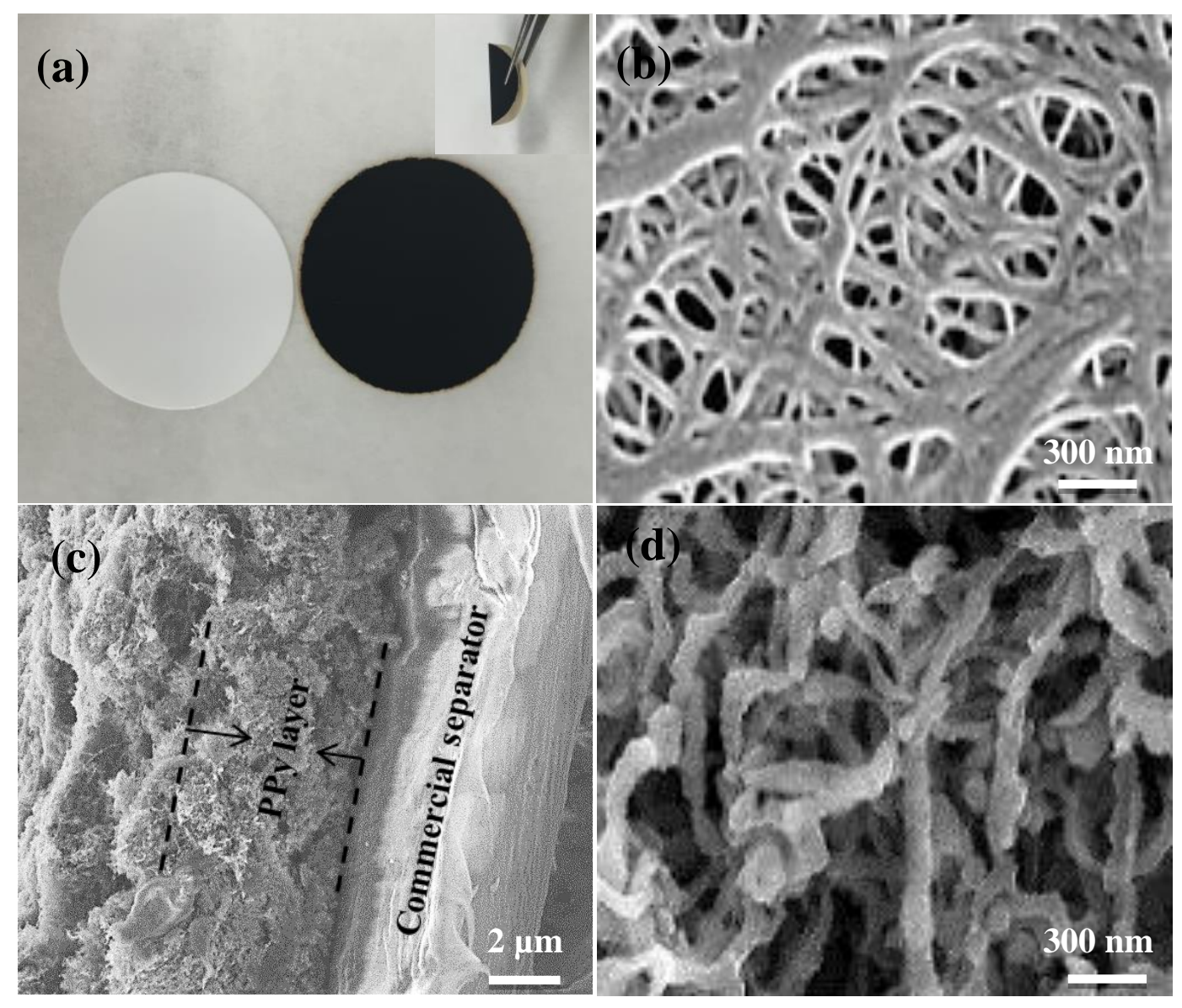

Fig. 5. (a) Digital photograph comparing the commercial separator and the PPy nanofiber coated commercial separator, with the inset showing the flexibility of the PPy nanofiber coated separator; (b) Top-view SEM image of commercial separator; (c) Cross-sectional SEM image of the PPy nanofiber coated separator; (d) Top-view SEM image of the PPy coated separator. 

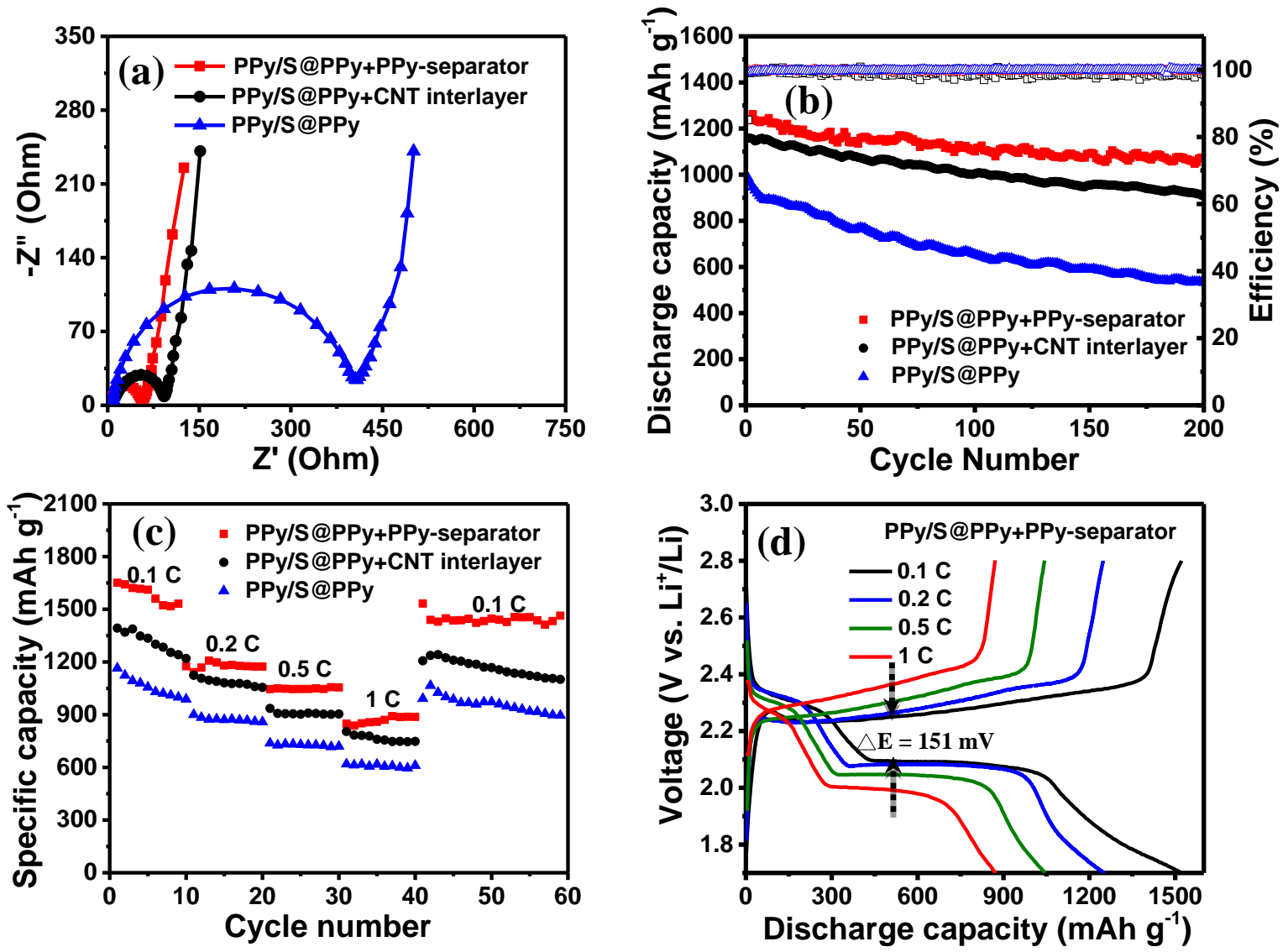

Fig. 6. (a) Nyquist plots of Li-S batteries with PPy/S@PPy, PPy/S@PPy+PPy-separator, and PPy/S@PPy+ CNT interlayer structure before cycling; (b) Cycling performance (0.2 C) and (c) rate performance of Li-S batteries with PPy/S@PPy, PPy/S@PPy+PPy-separator, and PPy/S@PPy+ CNT interlayer structure; (d) Discharge-charge profiles of Li-S batteries with PPy/S@PPy+PPy-separator structure at different current densities. 


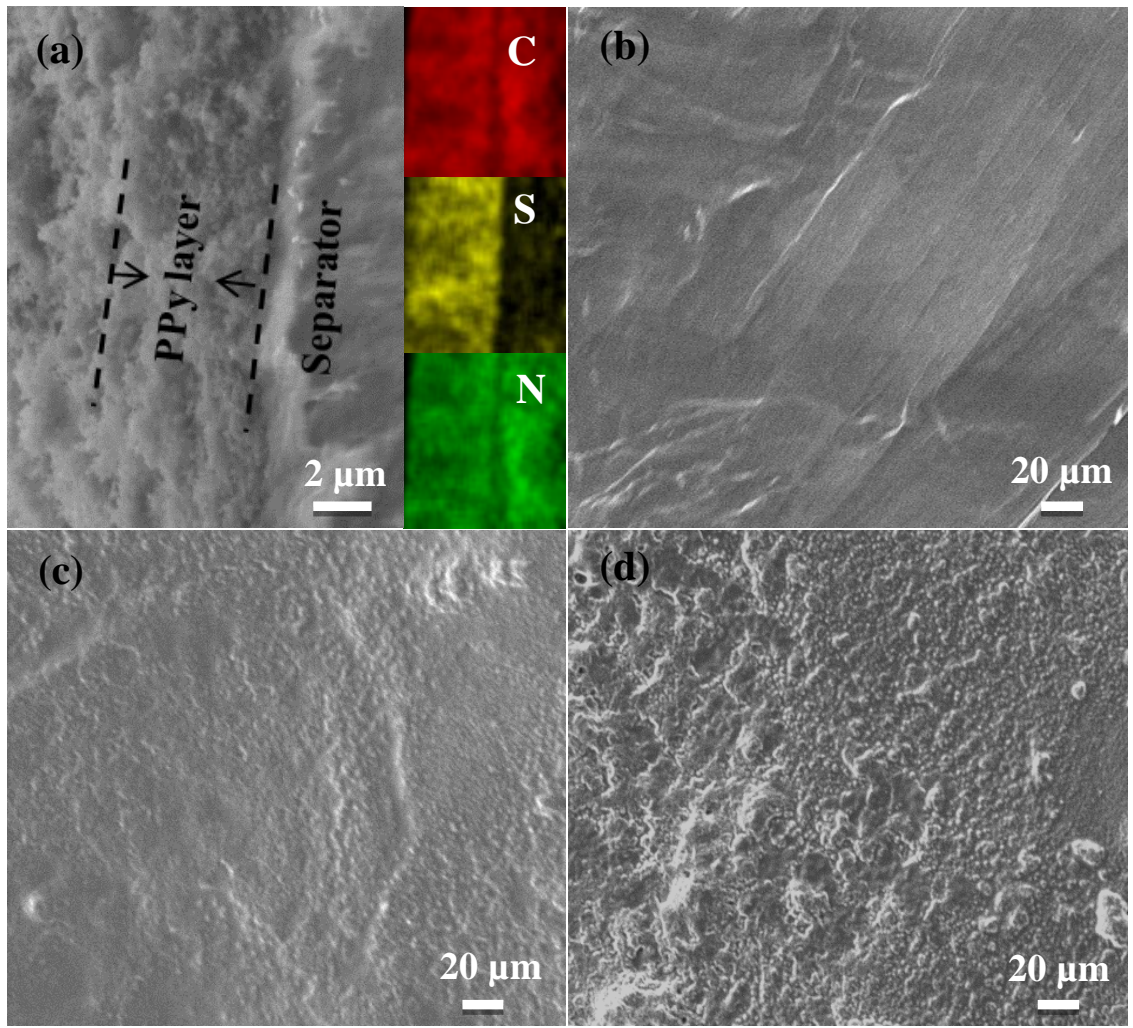

Fig. 7. (a) SEM image and element mappings for PPy coated separator after 50 cycles; SEM images of (b) pristine lithium, and lithium anodes from the batteries with PPy/S@PPy electrode (c) with and (d) without the PPy-coated separator after 50 cycles. 
(a) $\mathrm{Al} / \mathrm{S} @$ PPy cathode $\begin{aligned} & \text { Commercial } \\ & \text { separator }\end{aligned} \mathrm{Li}_{2} \mathrm{~S} / \mathrm{Li}_{2} \mathrm{~S}_{2}$

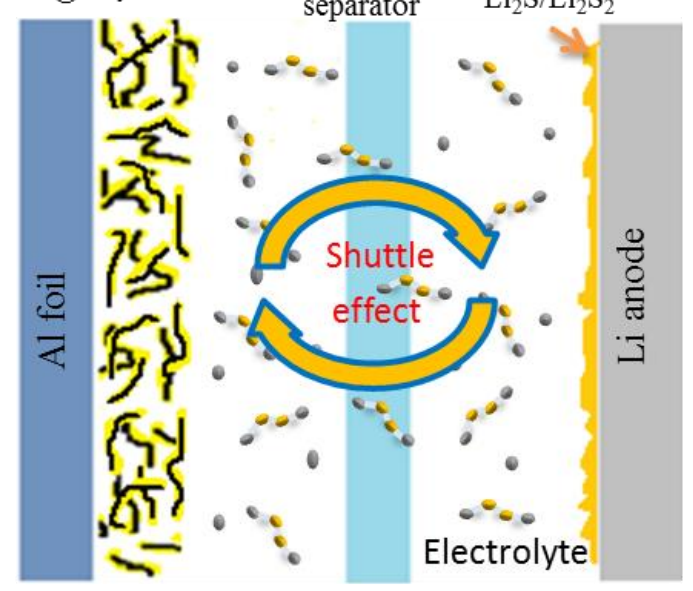

(b) PPy/S@PPy cathode

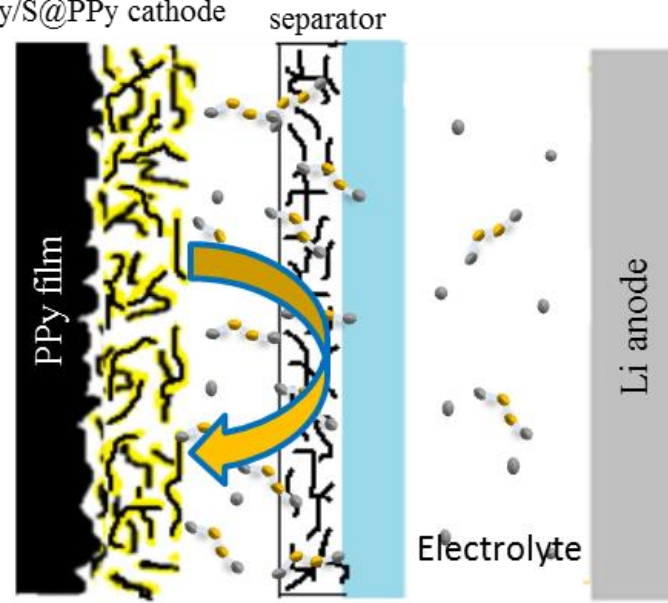

$\sim_{\text {s@PPy composites }}$

Lithium ion $\bullet \begin{aligned} & \text { Lithium } \\ & \text { polysulfides }\end{aligned}$

Fig. 8. Schematic illustration of $\mathrm{Li}-\mathrm{S}$ batteries with (a) the traditional electrode structure and

(b) the uniquely designed electrode structure. 

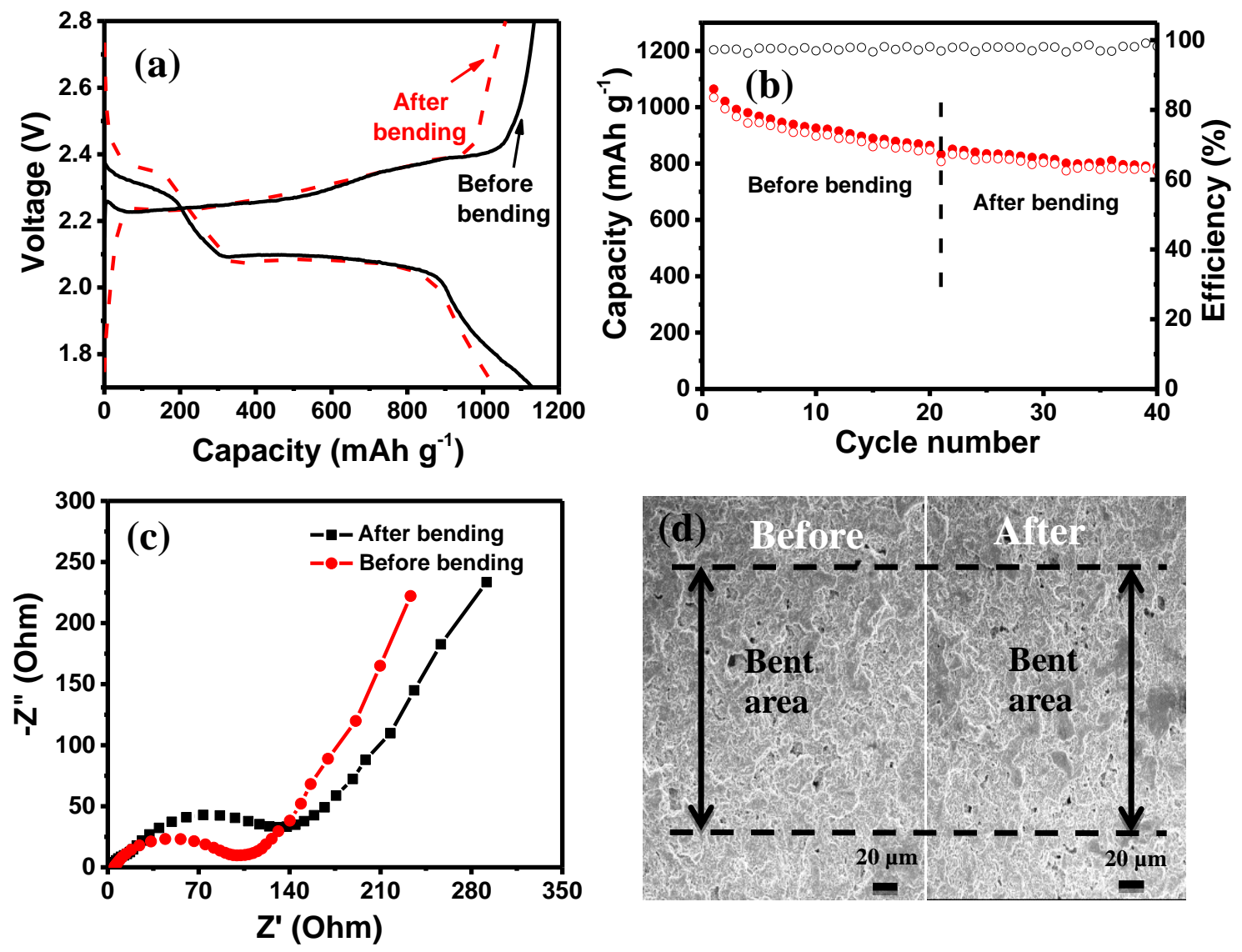

(e)

(f)
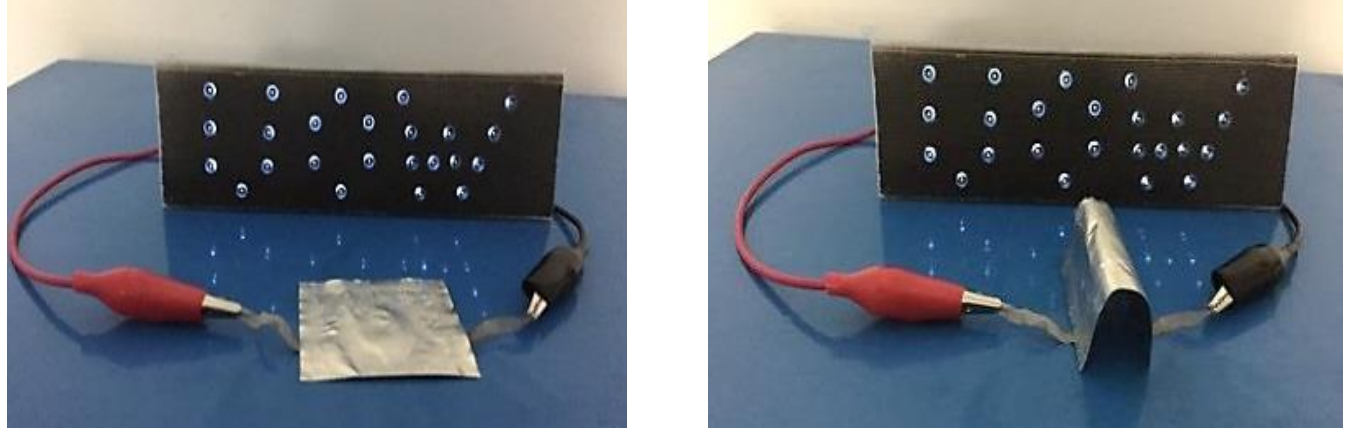

Fig. 9. (a) Charge/discharge curves of the soft-packaged battery before and after bending; (b) Cycling performance of the soft-packaged battery at $0.1 \mathrm{C}$; (c) Nyquist plots of the softpackaged battery before and after bending; (d) Field emission SEM images of the electrode before and after repeated-bending tests; The optical images show a white LED logo powered by a Li-S battery with a PPy/S@PPy+PPy-separator structure (e) before and (f) after bending. 